Review Article

\title{
Smoking Exposure and Survival of Patients with Esophagus Cancer: A Systematic Review and Meta-Analysis
}

\author{
Jun-jie Kuang, Zhi-min Jiang, Yan-xian Chen, Wei-peng Ye, Qiong Yang, \\ Hui-zhong Wang, and De-rong Xie \\ Department of Oncology, Sun Yat-sen Memorial Hospital, Sun Yat-sen University, Guangzhou, Guangdong 510120, China \\ Correspondence should be addressed to De-rong Xie; xiederong@21cn.com
}

Received 25 November 2015; Revised 28 February 2016; Accepted 29 February 2016

Academic Editor: Amosy M’Koma

Copyright (c) 2016 Jun-jie Kuang et al. This is an open access article distributed under the Creative Commons Attribution License, which permits unrestricted use, distribution, and reproduction in any medium, provided the original work is properly cited.

\begin{abstract}
Smoking is a well-known major risk factor in development of esophageal cancer, but few studies have reported the association between smoking status and prognosis of these patients. We conduct the present study to summarize current evidence. A computerized search of the PubMed and EMBASE was performed up to April 30, 2015. Eight studies, containing 4,286 patients, were analyzed. In the grouping analysis, among esophageal squamous-cell carcinoma patients, current and former smokers, compared to those who have never smoked, seemed to have a poorer prognosis ( $\mathrm{HR}=1.41,95 \% \mathrm{CI} 1.22-1.64$, and $\mathrm{HR}=1.35$, 95\% CI $0.92-$ 1.97 , resp.). In the subgroup analysis, adverse effects on current smoker compared with never smoker were also observed in China and the other countries ( $\mathrm{HR}=1.5,95 \% \mathrm{CI} 1.18-1.92$, and $\mathrm{HR}=1.36,95 \% \mathrm{CI} 1.12-1.65$, resp.). In the group that ever smoked, we could not get a similar result. No significantly increased risk was found in esophageal adenocarcinoma patients compared to the squamous-cell histology ones. In the smoking intensity analysis, heavy smoking was associated with poor survival in esophageal squamous-cell carcinoma. Our pooled results supported the existence of harmful effects of smoking on survival after esophagus cancer diagnosis.
\end{abstract}

\section{Introduction}

Esophageal cancer (EC) is the sixth leading cause of cancer mortality in the world. According to the official statistics of the US, about more than 18,000 cases were newly diagnosed and 15,000 deaths from EC in 2014, representing $5 \%$ of all cancer death [1]. Esophageal squamous-cell carcinoma (ESCC) and esophageal adenocarcinoma (EA) are the two main histological subtypes of esophagus cancer. Within recent decades, in some places, such as North America and Europe, the incidence and mortality rate of ESCC have decreased [2]. But in Asia, particularly in China, ESCC still occupies the vast majority of EC. Most patients with EC are already in locally advanced or metastatic disease at the time of diagnosis. With the lack of opportunity for radical surgery, radiation and chemotherapy became the major palliative treatment.

So far, sufficient population-based case-control and cohort studies have indicated that gastroesophageal reflux disease (GERD), cigarette smoking, and obesity are the main established risk factors for developing EC [3-8]. Studies have shown that, compared with nonsmokers, ESCC incidence risk is increased by approximately 3- to 7-fold in current smokers, and the risk of esophageal squamous-cell carcinoma is greater than adenocarcinoma [9]. But the relationship between smoking exposure and the prognosis of patients with esophagus cancer is still not clear. Thus, in this systematic review and meta-analysis, we perform a summary of literatures on the association between current, former, and never smoking behavior and survival of EC patients.

\section{Materials and Methods}

PubMed and EMBASE were searched to identify the related studies that had evaluated survival in association with smoking behavior in EC patients until 30 April, 2015. An effective search strategy was performed through keywords as follows: "smoking," "cigarette smoking," "tobacco smoking," "esophageal cancer," "esophageal squamous cell carcinoma," "esophageal adenocarcinoma," "oesophageal cancer," "oesophageal tumor," "gastro-esophageal junction cancer," 
"prognosis," and "survival". In addition, the reference lists of relevant articles were manually searched to find any other potentially eligible articles.

2.1. Selection Criteria. Case-control studies or cohort studies about the relationship between smoking status and oncological prognosis of patients with EC or gastroesophageal junction cancer were eligible to be included. Articles about smoking intensity are also included in the present study. We excluded reviews, commentaries, articles from overlapping samples, conference abstracts, and articles printed in languages other than English. The impact of smoking on survival had to be quantified by effect index such as hazard ratios (HR) with 95\% confidence intervals (CI). Quality of each eligible study was also rated independently by two reviewers. Finally, meeting abstracts were excluded. Two reviewers independently screened the database for titles and abstracts. If either reviewer though that certain title or abstract met eligibility criteria, the full text of the article was retrieved.

2.2. Data Extraction. Two reviewers (Jun-jie Kuang and Zhimin Jiang) identified potentially relevant studies by screening titles and/or abstracts of all citations identified through the database search. The full manuscripts of all articles identified in the search were screened for eligibility criteria by two independent reviewers (Jun-jie Kuang and Zhi-min Jiang). Disagreements were resolved through discussion. When necessary, a senior professor (De-rong Xie) helped to reach a consensus with all investigators. When an article provided more than one estimate, we chose the one adjusted for the largest set of variables. If a study published several update results, only the latest one would be included and analyzed. According to Newcastle-Ottawa Scale (NOS), the quality of each included article was assessed. For each study, the following characteristics were extracted using a standardized form: first author, country, publication year, study design, collection time, follow-up time, cases and controls, gender of subjects, age, tumor stage, clear definition of smoking status, adjustment variables, histological type, HR, and 95\% CI (Tables 1 and 2). In case of missing data, we contacted the primary investigators through emails.

2.3. Statistical Analysis. In the present meta-analysis, survival is measured from diagnosis to all-cause death. Those studies of low credibility, less than 6 points (NOS score range from 0 to 9 points), were excluded from the final meta-analysis [18]. We extracted HRs and estimated their standard errors indirectly with the help of Review Manage 5.3 software. Statistical heterogeneity was evaluated by the $Q$ test [19]. For the $Q$ statistic, a $p$ value of less than 0.10 was considered statistically significant heterogeneity. If heterogeneity was detected $(p<$ 0.10 ), random effects model was used; otherwise, fixed-effects model was used. Potential publication bias was identified by Begg's test and funnel plots [20]. Subgroup analysis based on geographical regions was also performed. All statistical analyses were carried out with Review Manage 5.3 version 5.3.3 (Cochrane Collaboration, Oxford, UK) and Stata version 12.0 (StataCorp, College Station, TX, USA) [21, 22].

\section{Results}

3.1. Study Characteristics. A total of 672 articles were obtained through initial search. Of those, 660 were excluded by title and abstract scan and another five by full-text reading. One additional article was found in the references. Finally, eight studies [10-17] were eligible for a qualitative analysis, including one cohort study [14] and seven case-control studies [10-13, 15-17] (Figure 1). Reviewers had perfect agreement in selecting the eight studies using the stated eligibility criteria. The eight studies comprise data from a total of 4,286 cases that were published between 2005 and 2013. Three studies were carried out in China $[10,13,14]$, and the other five studies were each from other countries (USA [11], Japan [12], Iran [15], Australia [16], and Sweden [17]). Sample size for the included studies ranged from 79 to 1,142 patients. Six studies [12-17] adjusted their measure for at least age, gender, Performance Status index, alcohol consumption, education, tumor stages, surgery history, nonsteroidal antiinflammatory drugs (NSAIDs), or body mass index (BMI). Six articles provided a definition of smoking [11-14, 16, 17]. Three studies analyzed the relationship between smoking intensity and prognosis $[12,14,16]$. Two of them provided HR values of mortality rate $[12,16]$. All studies gathered smoking status at the time of diagnosis. The main characteristics of the included studies are summarized in Table 1.

3.2. Systematic Review and Meta-Analysis. As we mentioned above, eight studies were eligible for the systematic review and meta-analysis (Table 3). In view of few survival data that had been found directly comparing with current smoker and former smoker, we divided the studies into three groups: current versus never smokers, former versus never smokers, and ever versus never smokers (Table 2). However, only two of the eight matched studies have contained an analysis of the survival of esophageal adenocarcinoma patients [11, 17]: one in both current versus never smoker and former versus never smoker groups $(\mathrm{HR}=1.0,95 \% \mathrm{CI} 0.6-1.7$, and $\mathrm{HR}=$ $0.9,95 \%$ CI $0.6-1.4$, resp.) [17] and the other one in the ever versus never smoker group $(\mathrm{HR}=0.86,95 \% \mathrm{CI} 0.64-1.16)$ [11]. No significantly increased risk of death was found in those two articles. With the lack of enough data on the survival of EA patients, the following results mainly aimed at the ESCC patients.

3.3. Current versus Never Smokers. All five trials analyzed the survival of current and never smokers [10, 13, 15-17]. The $I^{2}$ was $0 \%$ indicating that there was no heterogeneity in the pooled studies. Thus, in the fixed-effects model, current smokers showed a $41 \%$ higher mortality than never smokers ( $\mathrm{HR}=1.41,95 \%$ CI 1.22-1.64, $\left.p<0.00001, I^{2}=0 \%\right)$. A funnel plot of studies including survival of current and never smokers was created to estimate publication bias. It showed symmetric distribution, indicating that there may be minimal publication bias in the included studies. Begg's test showed no significant publication bias $(p=0.501)$. These analyses enhanced the reliability of our meta-analysis. See Figures 2 and 3. 


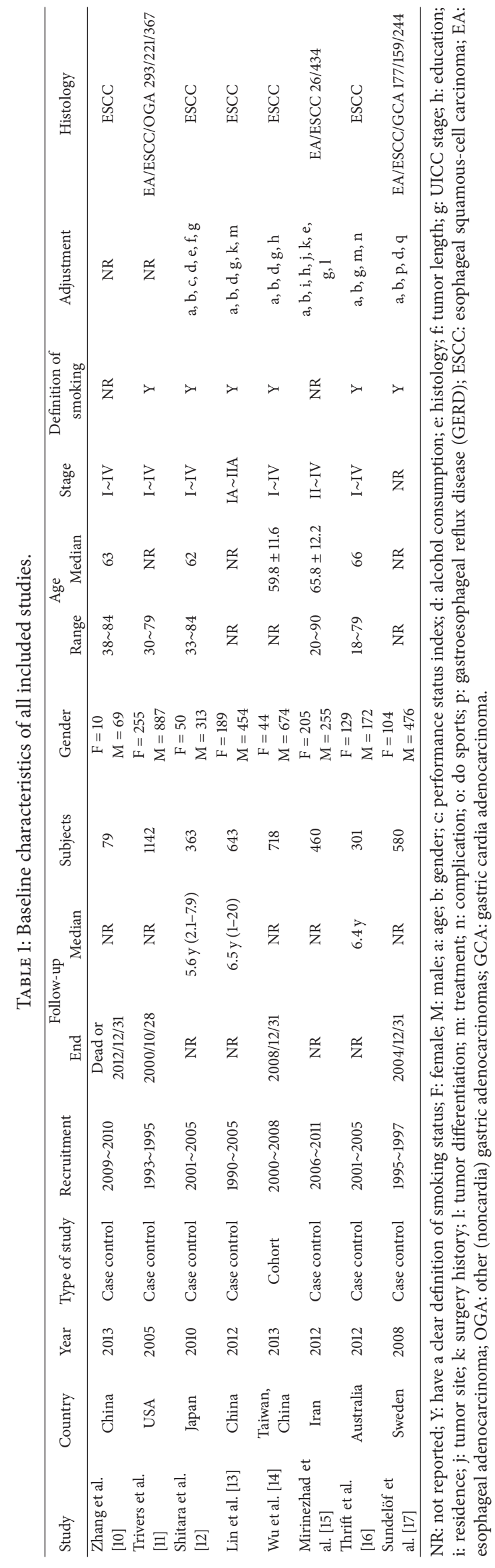




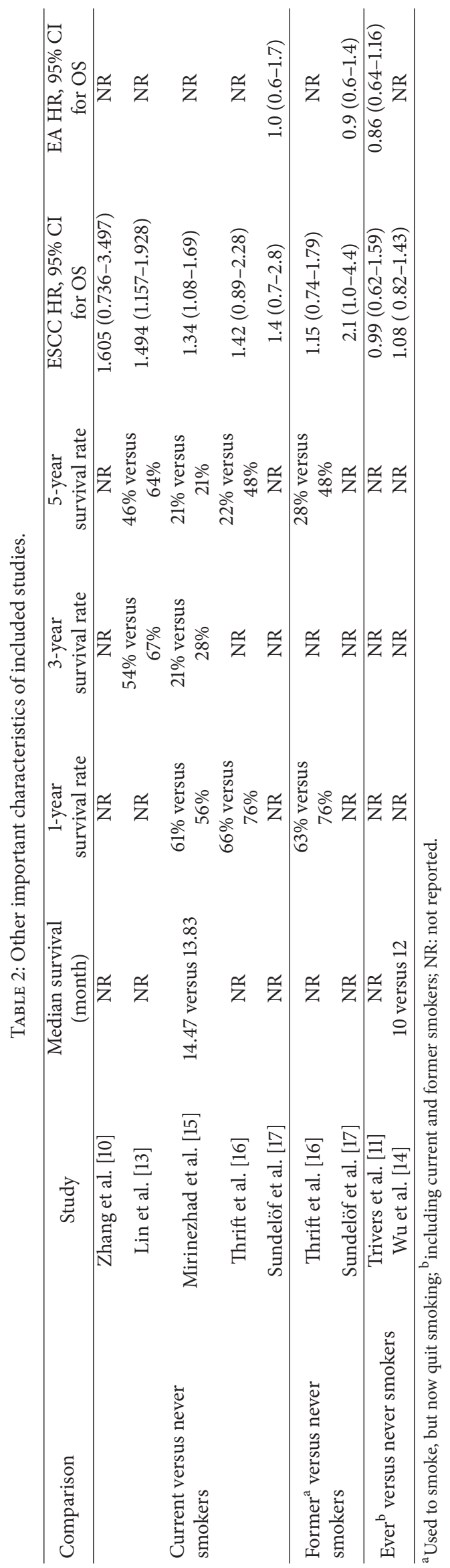




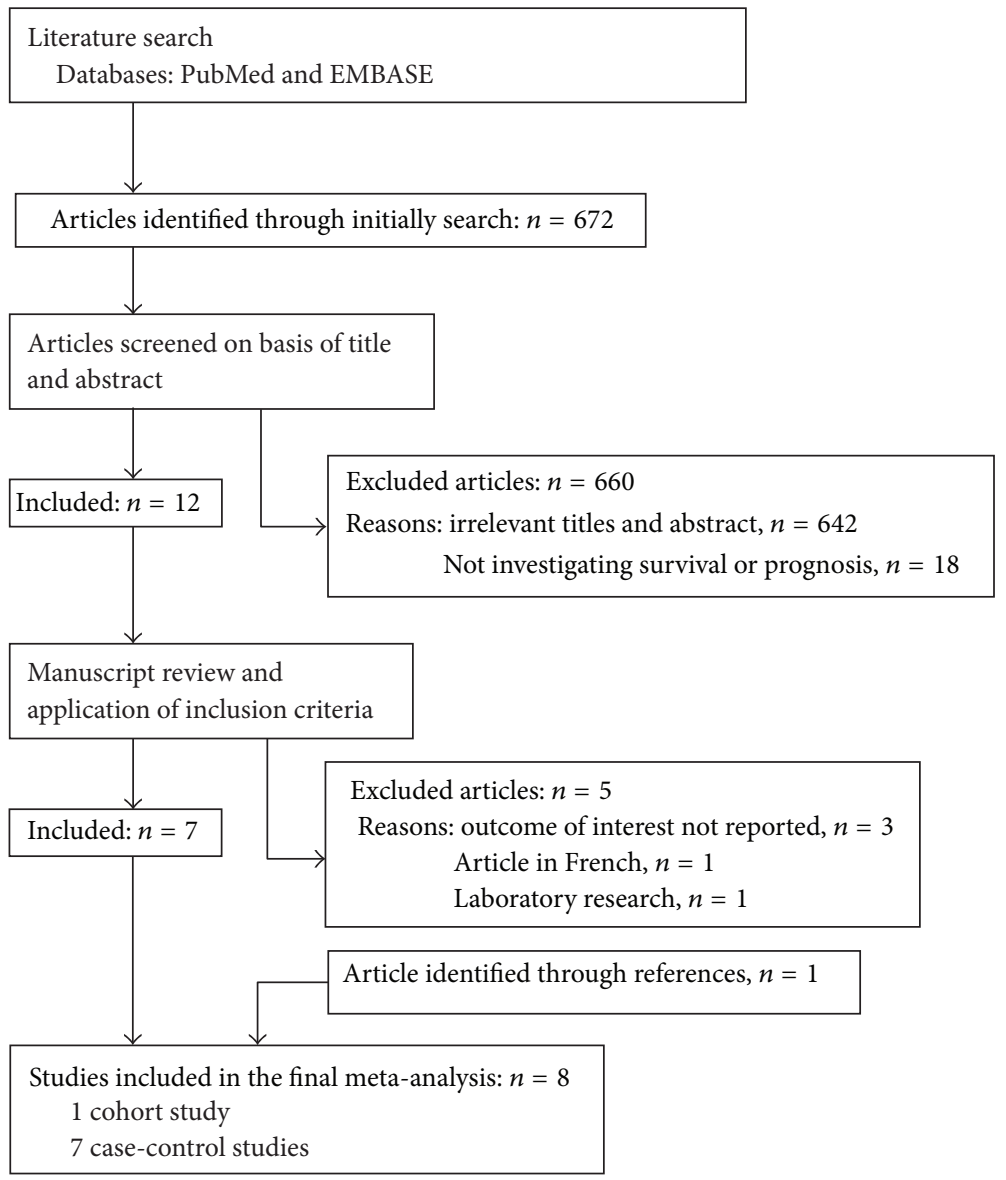

FIGURE 1: Flow chart of literature search for this meta-analysis.

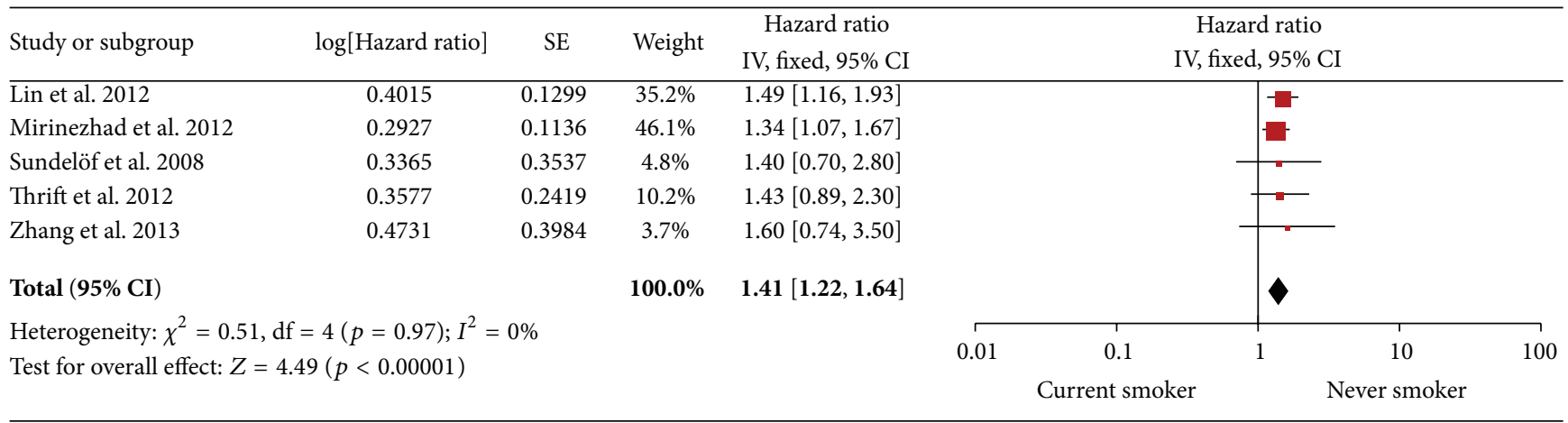

FIGURE 2: Current smoker versus never smoker.

In subgroup analysis, the adverse effects of current smoking on prognosis have been shown in China and other countries $(\mathrm{HR}=1.5,95 \% \mathrm{CI} 1.18-1.92$, and $\mathrm{HR}=1.36,95 \% \mathrm{CI}$ $1.12-1.65$, resp.). See Figure 4.

3.4. Former versus Never Smokers. Two studies reported the survival of former and never smokers [16, 17]. The result of the test for heterogeneity was also not significant $(p=0.42)$. The pooled estimate showed a HR of 1.35 (95\% CI 0.92-1.97, $p=0.17, I^{2}=47 \%$ ) in favor of patients who quit smoking. See Figure 5.
3.5. Ever versus Never Smokers. Two studies combined groups of current and former smokers in a category of ever smokers [11, 14]. However, no significant difference was found in survival between ever and never smokers in ESCC (HR = 1.07, 95\% CI 0.94-1.23, $\left.p=0.32, I^{2}=0 \%\right)$. See Figure 6.

3.6. Smoking Intensity. Three studies reported the relationship between survival and smoking intensity (Table 4) [12, 14, 16]. Two of them assessed intensity by smoking pack years (PY) and one by the cigarettes per day (C/D). In Shitara et al's study [12], $364 \mathrm{EC}$ patients were divided into two groups 
TABLE 3: Results of quality assessment by Newcastle-Ottawa Scale.

\begin{tabular}{l} 
Study \\
\hline Case control
\end{tabular}

TABLE 4: Mortality and survival of EC patients for different studies.

\begin{tabular}{|c|c|c|c|c|}
\hline \multirow{2}{*}{$\begin{array}{l}\text { Smoking intensity } \\
\text { (pack year PY or cigarette/day C/D) }\end{array}$} & \multirow{2}{*}{ Author } & \multirow{2}{*}{$\begin{array}{l}\text { Mean survival } \\
\text { time (month) }\end{array}$} & \multicolumn{2}{|c|}{ Mortality rate } \\
\hline & & & HR & $95 \% \mathrm{CI}$ \\
\hline $\mathrm{PY}<20$ & \multirow{4}{*}{ Shitara et al. [12] } & & 1 & - \\
\hline $20 \leqslant P Y$ & & & 1.73 & $(1.12-2.68)$ \\
\hline $20 \leqslant P Y<40$ & & & 1.77 & $(1.09-2.89)$ \\
\hline $40 \leqslant P Y$ & & & 1.69 & $(1.06-2.67)$ \\
\hline Never smoked & \multirow{4}{*}{ Thrift et al. [16] } & & 1 & - \\
\hline $0<\mathrm{PY}<15$ & & & 1.44 & $(0.89-2.31)$ \\
\hline $15 \leqslant \mathrm{PY}<30$ & & & 0.99 & $(0.59-1.65)$ \\
\hline $30 \leqslant P Y$ & & & 1.26 & $(0.79-2.02)$ \\
\hline $20 \geqslant C / D$ & \multirow[b]{2}{*}{ Wu et al. [14] } & $20.6 \pm 27.0$ & & \\
\hline $\mathrm{C} / \mathrm{D}>20$ & & $\begin{array}{c}19.1 \pm 25.6 \\
p=0.03\end{array}$ & & \\
\hline
\end{tabular}

PY: pack years; C/D: cigarettes per day.

according to the smoking status: nonheavy smokers $(\mathrm{PY}<20)$ versus heavy smokers $(P Y \geqslant 20)$. The 3 - and 5-year survival rates were likely lower in the heavy smoking group, especially in patients treated by chemoradiotherapy $(\mathrm{HR}=2.43,95 \% \mathrm{CI}$ 1.38-4.27, $p=0.002$ ). When the researchers further divided heavy smokers into two subgroups according to PY $(20<$ PY $<40$ and PY $>40$ ), the dose-response relationship was suggestive but not statistically significant in the multivariate analyses. No trend of increasing risk in early death was found across consumption categories $(p$-trend $=0.41$ and $p=0.53$ ) in other two studies. See Table 4.

\section{Discussion}

In clinical practice, we found that nearly $20 \sim 30 \%$ cancer patients were addicted to cigarettes, and many cancer survivors continue to smoke even after diagnosis [23]. Smoking is a well-known risk factor for esophageal cancer [23-25]. Yet, there are few studies that directly evaluate smoking as a prognostic factor for esophageal cancer [26]. To our knowledge, this is the first quantitative systematic review and meta-analysis on this topic, based on seven case-control studies [10-13, 15-17] and one cohort study [14] involving 


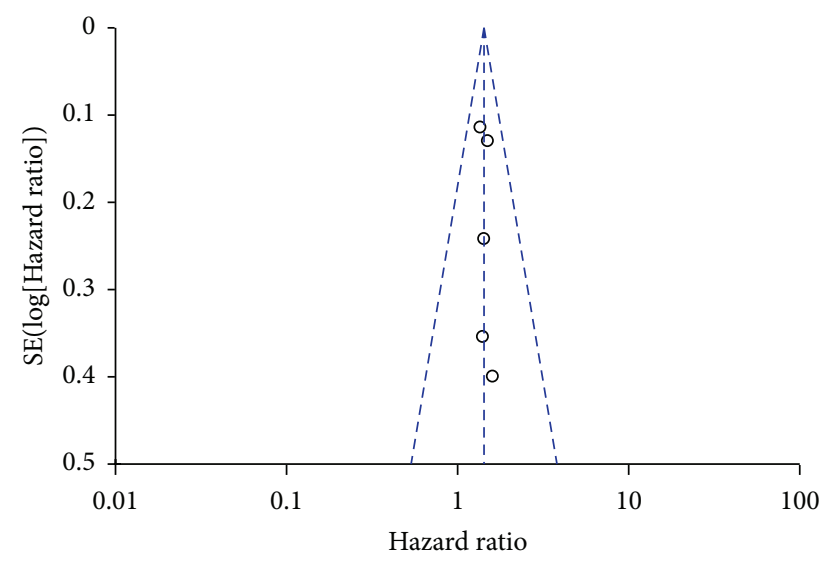

FIGURE 3: The funnel plot of current versus never smoker.

more than 4,000 cases. Only two of the eight matched studies have contained an analysis of the survival of esophageal adenocarcinoma patients $[11,17]$. Due to lack of sufficient data on the survival of EA patients, our results are mainly targeted at the ESCC patients.

An increased risk of $41 \%$ and $35 \%$ was estimated for current and former smokers compared to never smokers, respectively, even though the latter one was not statistically significant. When stratifying studies according to geographic areas, an adverse effect of current compared with never smoking was observed in China and other countries (Iran, Australia, and Sweden). The pooled HRs of two subgroups are very close $(\mathrm{HR}=1.5,95 \% \mathrm{CI} 1.18-1.92$, and $\mathrm{HR}=1.36,95 \%$ CI 1.12-1.65, resp.). These findings indicate that geographical difference would not interfere with the reliability of this meta-analysis. Unfortunately, neither Zhang et al.s [10] nor Mirinezhad et al.s [15] studies provide a clear definition of smoking status. Since a cigarette smoker may not just be a current smoker, we conduct a sensitivity analysis which excluded these two studies in the current smoking analysis. The conclusions were not altered in sensitivity analyses (HR $=1.47,95 \%$ CI $1.19-1.82, p=0.0004$ ).

We fail to replicate the similar result in the ever smoker group $(\mathrm{HR}=1.07,95 \%$ CI $0.94-1.23)$. It may be due to a different definition of smoking status. For example, Sundelöf et al's study [17] defined former smoking as having quit smoking for at least two years and ever smoking as individuals smoking regularly at least one cigarette per day or at least one cigar or pipe per week during a period of at least six months, while in Shitara et al.s study [12] former smokers were defined as those who quit smoking at least 1 year before the survey.

Looking at smoking intensity, we found significantly worse survival rates in esophageal squamous-cell cancer patients with a history of heavy tobacco smoking (PY of more than 15 or 20). But, in Shitara et al.'s study, the doseresponse relationship was not statistically significant when they divided heavy smokers into two groups according to PY $(20<\mathrm{PY}<40$ and PY $>40)$. Similar result can be found in Thrift et al.'s study [16], when PY of more than 15 was defined as heavy smoking. The difference between experimental and clinical observation findings may be attributed to the following reasons. Firstly, no consensus on the threshold of heavy smoking has been reached around the world. Secondly, all the collected studies gathered smoking history prior to the diagnosis and did not evaluate the impact of behavioral change in smoking after diagnosis, which might also be an interfering factor. To our knowledge, a substantial number of studies have applied this value (PY 20 or Brinkman index of $400)$ as the threshold of heavy smoking [12, 27-29]. Also, in NCCN Guideline, individuals (age $\geqslant 50$ y) with more than 20 PY history of smoking tobacco are selected as high risk group for lung cancer [30]. Due to the lack of consensus on the threshold of heavy smoking, Shitara et al.s [12] point might be more reasonable.

Overall, our review suggests that smoking prevention and cessation would be beneficial for prolonging EC patients' survival. ASCO Tobacco Cessation Guideline published in 2015 (version 1) also recommend that patients with cancer who continue to use tobacco have poorer treatment outcomes compared to their counterparts who do not use tobacco, regardless of whether the cancer was tobacco related [31].

Possible mechanisms linking tobacco exposure and EC outcomes are not yet completely clear. Taghavi et al. [32] found that overexpression of p53 in association with cigarette smoking may play a critical role in ESCC. In addition, p21 overexpression was found to be associated with poor prognosis, specifically in the operable ESCC patients. Moreover, Yamashita et al. [33] revealed that smoking might vary the activity of the 5-FU-related metabolic enzymes, resulting in poor curative effect. 5-Fluorouracil (5-FU) is an anticancer agent widely used in the treatment of digestive tract tumors. Yang et al. [34] showed that smoking can also cause tumor hypoxia, affecting its sensitivity to chemotherapy. So, we speculate that smoking not only is inducing malignant transformation of normal cells but may also change tumor cell gene or related metabolic activity and thus make tumor cells more aggressive and have poorer sensitivity to radiotherapy and chemotherapy. Furthermore, given that smoking causes other complications, part of the excess risk of smoking is that EC patients may succumb to cardiovascular or respiratory disease. Thrift et al. [16] indicated that effects on EC-specific mortality might be driven by some certain characteristics, such as age, sex, pretreatment AJCC tumor stage, treatment, and presence of comorbidities ( $\mathrm{HR}=1.34,95 \% \mathrm{CI} 1.02-1.75)$.

Our study points to areas of research that need more attention to enable a more complete understanding of the role in EC survival and the potential to enhance survival of EC patients by smoking prevention and cessation.

This review and meta-analysis has limitations. First, neither Zhang et al.s [10] nor Mirinezhad et al.s [15] studies provide a clear definition of smoking status. A cigarette smoker may not just be a current smoker. Second, based on the predetermined search strategy, we cannot rule out the possibility of having missed relevant articles, especially when the articles are written in languages other than English. Third, no enough information was found on the relationship between smoking and the survival of esophageal junction cancer or esophageal adenocarcinoma; our conclusions mainly focus on the prognosis of ESCC patients. Fourth, meaningful meta-analyses could only be carried out on 


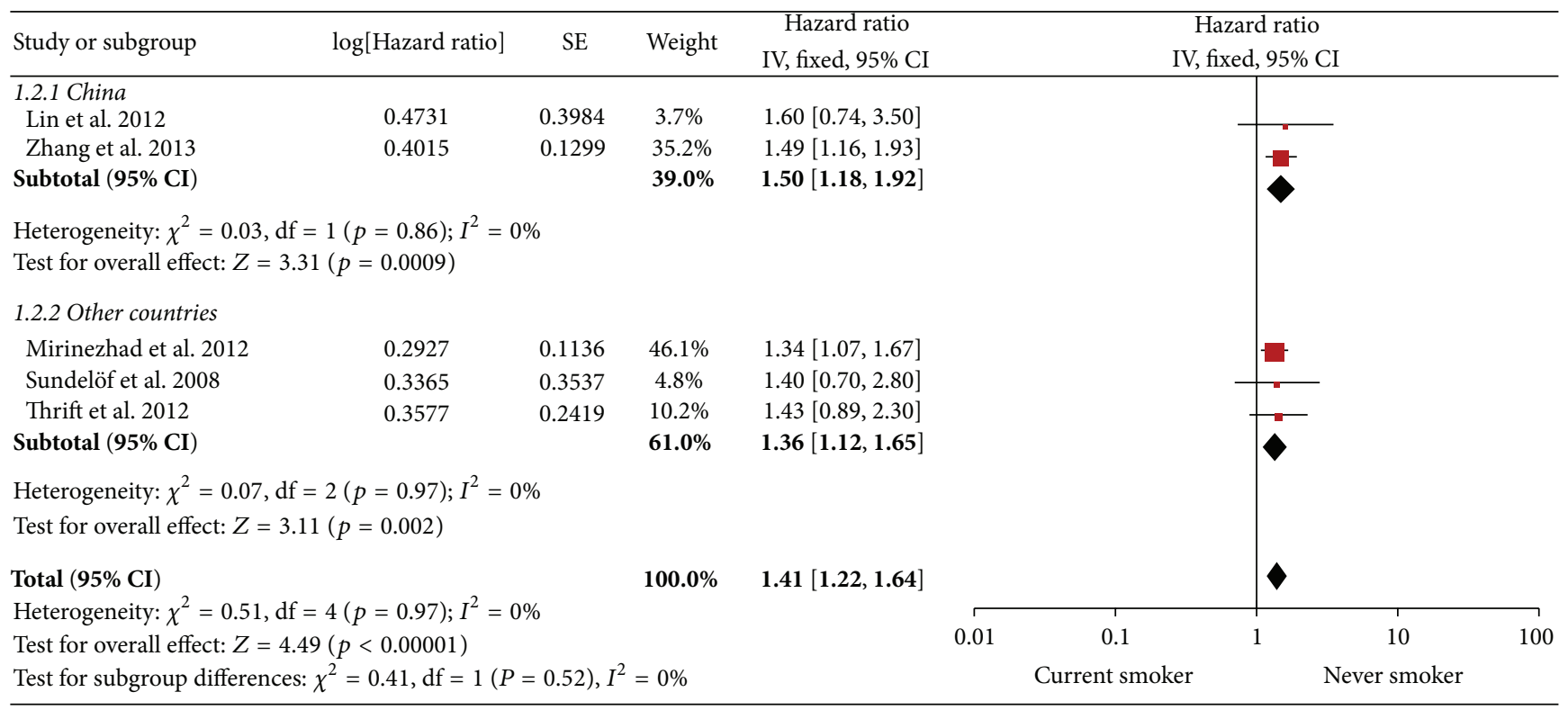

FIGURE 4: Subgroup analysis of current versus never smoker.

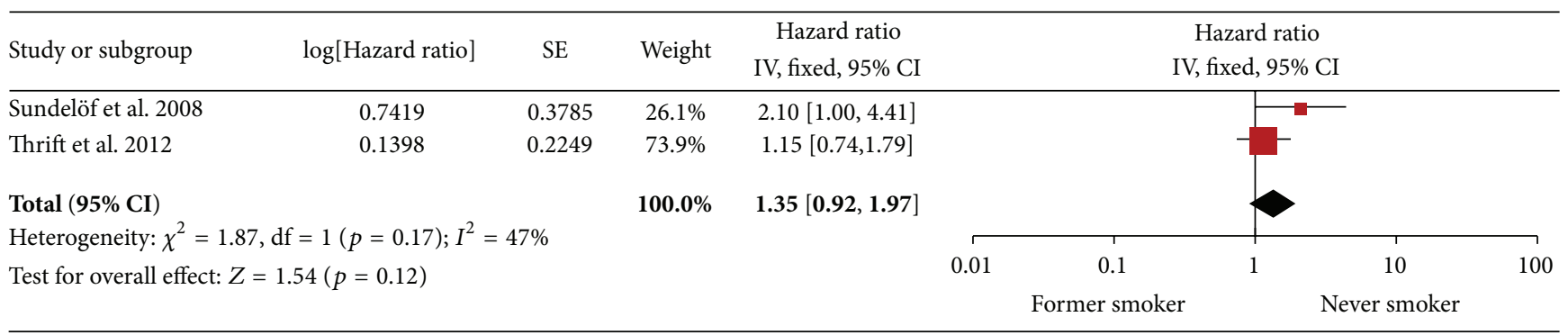

FIGURE 5: Former versus never smoker.

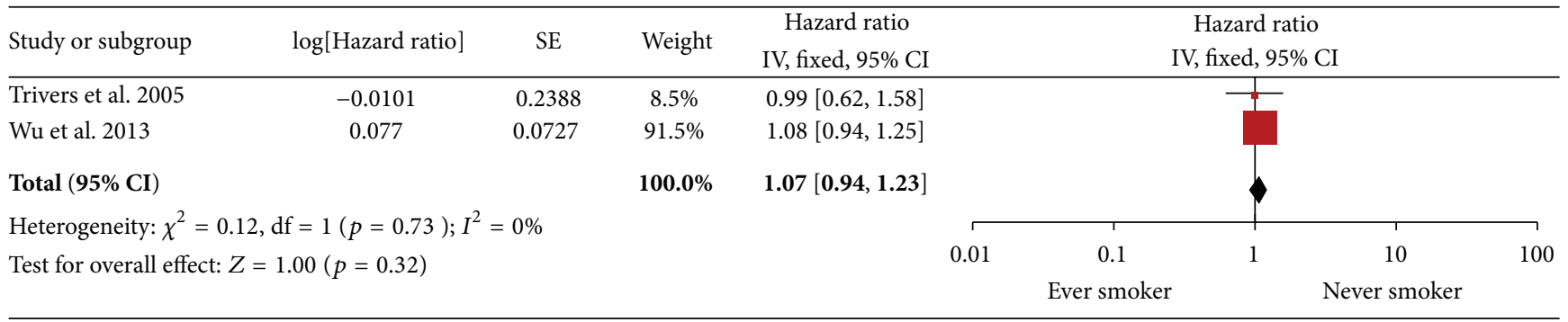

FIGURE 6: Ever versus never smoker.

the increased mortality for current and former smokers compared with never smokers. Comparability was interfered by various definitions and categorizations of smoking exposure, heterogeneity in inclusion and exclusion criteria, and covariates adjusted for [35]. Fifth, we combined results from cohort studies and case-control studies together in the metaanalysis. Strictly speaking, it is not very appropriate. But there are too few articles included in the ever versus never smokers group analysis. Similar statistical methods could be found in an article published in Journal of Clinical Oncology [36]. Moreover, worried about taking blame, some EC patients may refuse to provide real smoking history when visiting their physicians. Finally, there may have been negative studies that were never published, even though there was no indication of publication bias in our meta-analyses.

\section{Conclusions}

In summary, our pooled results support the existence of harmful effects of smoking on survival even after esophagus cancer diagnosis. Tobacco control and smoking cessation should be considered as an important part of a long-term 
treatment of esophagus cancer. Large population-based and well-designed studies are needed to further clarify the benefit of smoking prevention and cessation for EC patients.

\section{Competing Interests}

The authors declare that they have no competing interests.

\section{Authors' Contributions}

Jun-jie Kuang and Zhi-min Jiang contributed equally to this work so that they should be considered as the cofirst authors. Jun-jie Kuang and De-rong Xie conceive and design the experiments. Jun-jie Kuang and Zhi-min Jiang collected data and performed statistical analyses, Jun-jie Kuang drafted the paper, De-rong Xie, Qiong Yang, Wei-peng Ye, Yan-xian Chen, and Hui-zhong Wang revised the paper and contributed to the discussion, and all authors read and approved the final paper.

\section{Acknowledgments}

The authors of this paper would like to thank the authors who kindly provided the data necessary for the study.

\section{References}

[1] A. K. Rustgi and H. B. El-Serag, "Esophageal carcinoma," The New England Journal of Medicine, vol. 371, no. 26, pp. 24992509, 2014.

[2] J. H. Rubenstein and N. J. Shaheen, "Epidemiology, diagnosis, and management of esophageal adenocarcinoma," Gastroenterology, vol. 149, no. 2, pp. 302-317.e1, 2015.

[3] Y. Kinjo, Y. Cui, S. Akiba et al., "Mortality risks of oesophageal cancer associated with hot tea, alcohol, tobacco and diet in Japan,” Journal of Epidemiology, vol. 8, no. 4, pp. 235-243, 1998.

[4] K. K. Cheng, N. E. Day, and T. W. Davies, "Oesophageal cancer mortality in Europe: paradoxical time trend in relation to smoking and drinking," British Journal of Cancer, vol. 65, no. 4, pp. 613-617, 1992.

[5] A. Cayuela, J. Vioque, and F. Bolumar, "Oesophageal cancer mortality: relationship with alcohol intake and cigarette smoking in Spain," Journal of Epidemiology and Community Health, vol. 45, no. 4, pp. 273-276, 1991.

[6] K. Sakata, Y. Hoshiyama, S. Morioka et al., "Smoking, alcohol drinking and esophageal cancer: findings from the JACC Study," Journal of Epidemiology, vol. 15, supplement 2, pp. S212-S219, 2005.

[7] M. Karam-Hage, P. M. Cinciripini, and E. R. Gritz, "Tobacco use and cessation for cancer survivors: an overview for clinicians," CA: A Cancer Journal for Clinicians, vol. 64, no. 4, pp. 272-290, 2014.

[8] P. J. Veugelers, G. A. Porter, D. L. Guernsey, and A. G. Casson, "Obesity and lifestyle risk factors for gastroesophageal reflux disease, Barrett esophagus and esophageal adenocarcinoma," Diseases of the Esophagus, vol. 19, no. 5, pp. 321-328, 2006.

[9] F. Kamangar, W.-H. Chow, C. C. Abnet, and S. M. Dawsey, "Environmental causes of esophageal cancer," Gastroenterology Clinics of North America, vol. 38, no. 1, pp. 27-57, 2009.
[10] F. Zhang, H. Han, C. Wang et al., "A retrospective study: the prognostic value of anemia, smoking and drinking in esophageal squamous cell carcinoma with primary radiotherapy," World Journal of Surgical Oncology, vol. 11, article 249, 2013.

[11] K. F. Trivers, A. J. De Roos, M. D. Gammon et al., "Demographic and lifestyle predictors of survival in patients with esophageal or gastric cancers," Clinical Gastroenterology and Hepatology, vol. 3, no. 3, pp. 225-230, 2005.

[12] K. Shitara, K. Matsuo, S. Hatooka et al., "Heavy smoking history interacts with chemoradiotherapy for esophageal cancer prognosis: a retrospective study," Cancer Science, vol. 101, no. 4, pp. 1001-1006, 2010.

[13] Y. Lin, X. Su, H. Su et al., "Prediagnostic smoking and postoperative survival in lymph node-negative esophagus squamous cell carcinoma patients," Cancer Science, vol. 103, no. 11, pp. 19851988, 2012.

[14] I.-C. Wu, C.-C. Wu, C.-Y. Lu et al., "Substance use (alcohol, areca nut and cigarette) is associated with poor prognosis of esophageal squamous cell carcinoma," PLoS ONE, vol. 8, no. 2, Article ID e55834, 2013.

[15] S. K. Mirinezhad, M. H. Somi, A. G. Jangjoo et al., "Survival rate and prognostic factors of esophageal cancer in East Azerbaijan province, North-west of Iran," Asian Pacific Journal of Cancer Prevention, vol. 13, no. 7, pp. 3451-3454, 2012.

[16] A. P. Thrift, C. M. Nagle, P. P. Fahey et al., “The influence of prediagnostic demographic and lifestyle factors on esophageal squamous cell carcinoma survival," International Journal of Cancer, vol. 131, no. 5, pp. E759-E768, 2012.

[17] M. Sundelöf, J. Lagergren, and W. Ye, "Patient demographics and lifestyle factors influencing long-term survival of oesophageal cancer and gastric cardia cancer in a nationwide study in Sweden," European Journal of Cancer, vol. 44, no. 11, pp. 1566-1571, 2008.

[18] http://www.ohri.ca/programs/clinical_epidemiology/oxford .asp.

[19] J. P. T. Higgins and S. G. Thompson, "Quantifying heterogeneity in a meta-analysis," Statistics in Medicine, vol. 21, no. 11, pp. 15391558, 2002.

[20] J. A. C. Sterne, M. Egger, and G. D. Smith, "Systematic reviews in health care: investigating and dealing with publication and other biases in meta-analysis," British Medical Journal, vol. 323, no. 7304, pp. 101-105, 2001.

[21] Cochrane IMS, http://tech.cochrane.org/revman/download.

[22] http://www.stata.com/order/.

[23] I. Ferronha, A. Bastos, and N. Lunet, "Prediagnosis lifestyle exposures and survival of patients with gastric cancer: systematic review and meta-analysis," European Journal of Cancer Prevention, vol. 21, no. 5, pp. 449-452, 2012.

[24] N. Pandeya, G. M. Williams, S. Sadhegi, A. C. Green, P. M. Webb, and D. C. Whiteman, "Associations of duration, intensity, and quantity of smoking with adenocarcinoma and squamous cell carcinoma of the esophagus," American Journal of Epidemiology, vol. 168, no. 1, pp. 105-114, 2008.

[25] S. Ishiguro, S. Sasazuki, M. Inoue, N. Kurahashi, M. Iwasaki, and S. Tsugane, "Effect of alcohol consumption, cigarette smoking and flushing response on esophageal cancer risk: a populationbased cohort study (JPHC study)," Cancer Letters, vol. 275, no. 2, pp. 240-246, 2009.

[26] P. P. Fahey, K.-A. Mallitt, T. Astell-Burt, G. Stone, and D. C. Whiteman, "Impact of pre-diagnosis behavior on risk of death from esophageal cancer: a systematic review and meta-analysis," Cancer Causes and Control, vol. 26, no. 10, pp. 1365-1373, 2015. 
[27] S. M. Park, M. K. Lim, S. A. Shin, and Y. H. Yun, "Impact of prediagnosis smoking, alcohol, obesity, and insulin resistance on survival in male cancer patients: National Health Insurance Corporation Study," Journal of Clinical Oncology, vol. 24, no. 31, pp. 5017-5024, 2006.

[28] A. I. Phipps, J. Baron, and P. A. Newcomb, "Prediagnostic smoking history, alcohol consumption, and colorectal cancer survival: the Seattle Colon Cancer Family Registry," Cancer, vol. 117, no. 21, pp. 4948-4957, 2011.

[29] N. J. McCleary, D. Niedzwiecki, D. Hollis et al., "Impact of smoking on patients with stage III colon cancer: results from cancer and leukemia group B 89803," Cancer, vol. 116, no. 4, pp. 957-966, 2010.

[30] http://www.nccn.org/professionals/physician_gls/f_guidelines .asp\#smoking_cessation.

[31] http://www.asco.org/practice-research/tobacco-cessation-andcontrol-resources.

[32] N. Taghavi, F. Biramijamal, M. Sotoudeh et al., "Association of p53/p21 expression with cigarette smoking and prognosis in esophageal squamous cell carcinoma patients," World Journal of Gastroenterology, vol. 16, no. 39, pp. 4958-4967, 2010.

[33] T. Yamashita, K. Kato, N. K. Long et al., "Effects of smoking and alcohol consumption on 5-fluorouracil-related metabolic enzymes in oral squamous cell carcinoma," Molecular and Clinical Oncology, vol. 2, no. 3, pp. 429-434, 2014.

[34] P.-Y. O. Yang, Z. Su, Y.-P. Mao et al., "Prognostic impact of cigarette smoking on the survival of patients with established nasopharyngeal carcinoma," Cancer Epidemiology Biomarkers \& Prevention, vol. 22, no. 12, pp. 2285-2294, 2013.

[35] V. Walter, L. Jansen, M. Hoffmeister, and H. Brenner, "Smoking and survival of colorectal cancer patients: systematic review and meta-analysis," Annals of Oncology, vol. 25, no. 8, pp. 1517-1525, 2014.

[36] S. Bonovas, K. Filioussi, N. Tsavaris, and N. M. Sitaras, "Use of statins and breast cancer: a meta-analysis of seven randomized clinical trials and nine observational studies," Journal of Clinical Oncology, vol. 23, no. 34, pp. 8606-8612, 2005. 


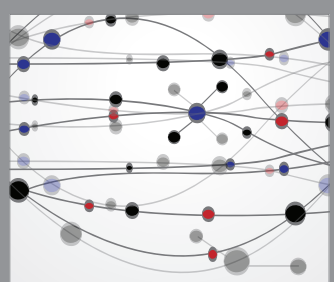

The Scientific World Journal
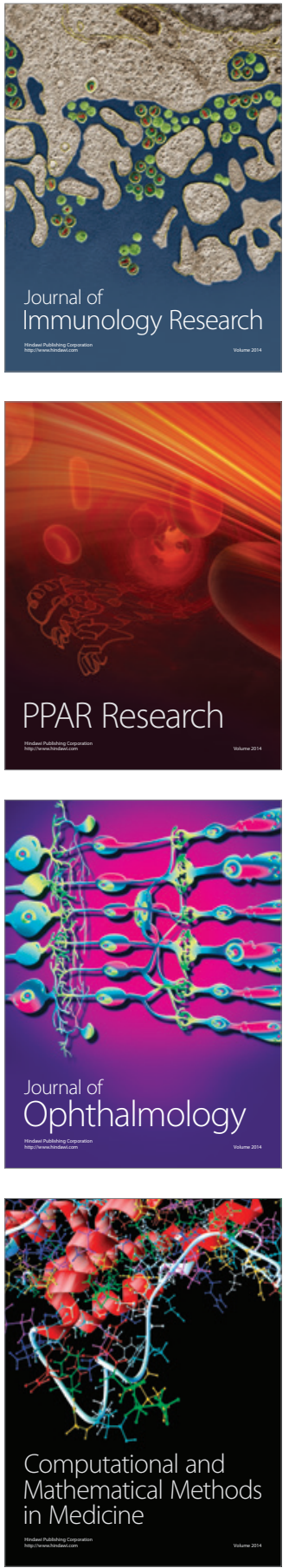

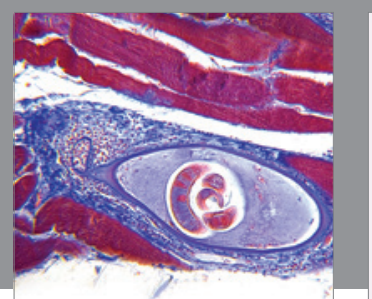

Gastroenterology Research and Practice

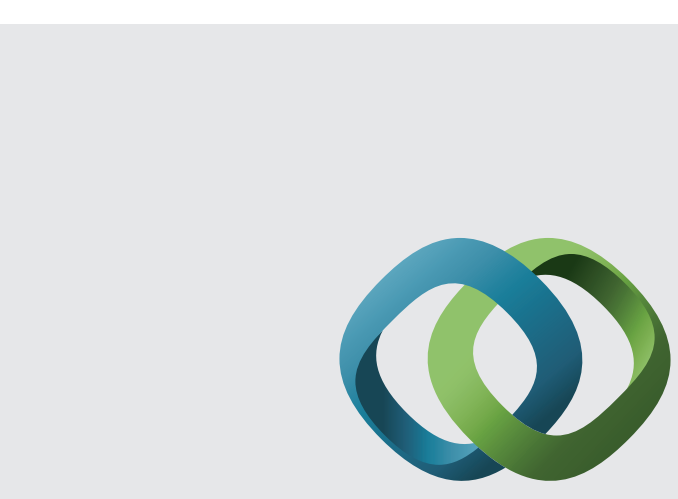

\section{Hindawi}

Submit your manuscripts at

http://www.hindawi.com
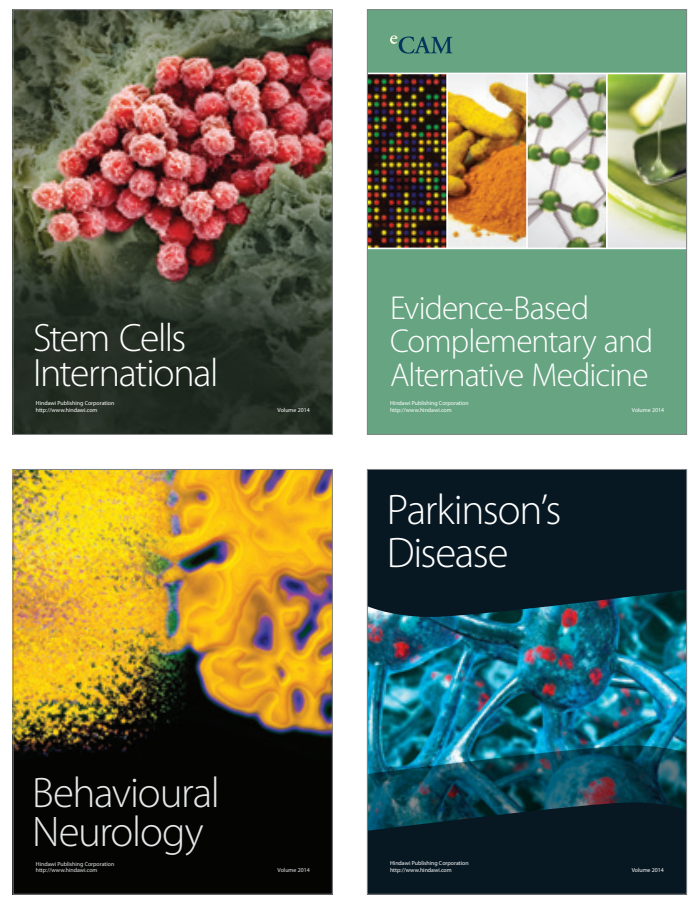
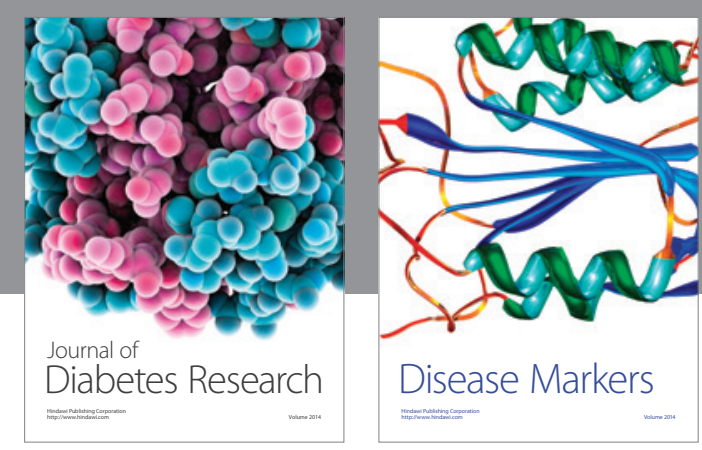

Disease Markers
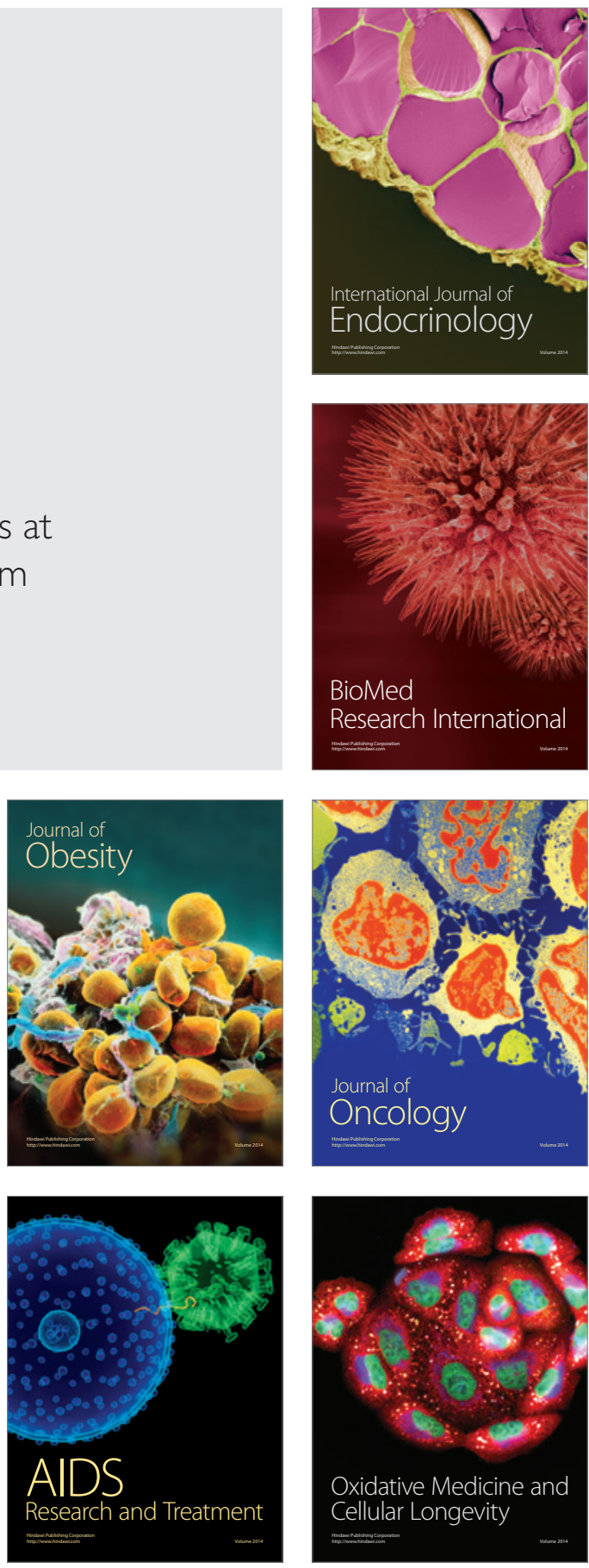